\title{
Sleep Disorders and Chronic Constipation: Relation to Other Co-morbidities?
}

\author{
S. L. Szeinbach ${ }^{*}, 1$, R. Rodriguez-Monguio ${ }^{2}$, R. W. Baran ${ }^{3}$ and P.B. Williams ${ }^{4}$ \\ ${ }^{1}$ Division of Pharmacy Practice \& Administration, College of Pharmacy, Ohio State University, Columbus, Ohio, USA \\ ${ }^{2}$ School of Pubic Health and Health Sciences, University of Massachusetts, Amherst, Massachusetts, USA and The Insti- \\ tute for Global Health, University of Massachusetts, Amherst, Massachusetts, USA \\ ${ }^{3}$ Global Health Economics, Abbott Laboratories, Abbott Park, Illinois, USA \\ ${ }^{4}$ Medical School, University of Missouri, Kansas City, MO, USA
}

\begin{abstract}
Sleep disorders are common complaints and frequently associated with a number of disease states. Although the link between sleep disorders, respiratory diseases, and other co-morbid conditions experienced by patients has been investigated, the link between sleep disorders and chronic constipation is relatively unexplored. Given the widespread occurrence of sleep disorders, it is important to evaluate how sleep disorders relate to respiratory diseases and other comorbid disease states in participants with chronic constipation. Thus, the purpose of this study was to identify co-morbid conditions that were significant predictors for chronic constipation participants with and without sleep disorders. Of the 311 participants with a confirmed diagnosis of chronic constipation, $84 \%$ of the sample was female with $52 \%$ having sleep disorders, approximately two-thirds reported allergies, and $30 \%$ or less reported chronic sinusitis, chronic bronchitis, asthma, and chronic obstructive pulmonary disease (COPD). Results from logistic regression analysis revealed significant odds ratios for sleep disorders and allergies (OR 2.4; $\mathrm{p}=0.001)$, asthma (OR 2.0; $\mathrm{p}=0.01$ ), chronic bronchitis (OR 2.2; $\mathrm{p}$ $=0.014)$, COPD (OR 4.0; $<<0.001)$, sinusitis (OR 2.0; $\mathrm{p}=0.008)$, and for other co-morbid conditions. Perceptions of overall health were significantly worse $(\mathrm{p}<0.001)$ for chronic constipation participants with certain co-morbid conditions including sleep disorders, respiratory diseases, and disease states producing pain. Co-morbid conditions may influence information provided by patients when discussing family history, diagnosing, discussing pharmacotherapy, and monitoring patient-reported outcomes. Co-morbid conditions should be considered by physicians and specialists during patient assessment and evaluation.
\end{abstract}

Keyword: Allergic disease, upper respiratory, insomnia, Constipation, Co-morbid conditions, diagnosis, clinical evaluation.

\section{INTRODUCTION}

Patient complaints are important considerations in diagnostic evaluation and are often used to reinforce information gleaned from diagnostic testing and other test procedures.

Within the scope of patient evaluation, some complaints are relatively easy to pinpoint and attribute to specific disease states. For example, respiratory diseases such as allergic rhinitis, asthma, chronic obstructive pulmonary disease, and sinusitis are known to trigger patient complaints related to poor sleep quality $[1,2]$. Although sleep disorders are common complaints associated with many disease states [3, 4], the relationship between sleep disorders, allergic diseases and other co-morbidities is relatively unexplored in patients with chronic constipation. Besides the influence of comorbidities on pharmacotherapy, co-morbidities also have a substantial impact on health care utilization.

The economic burden from co-morbid conditions is welldocumented. Sleep disorders were estimated to cost 30 to 35 billion US dollars annually in 1994, which included illness,

Address correspondence to this author at Division of Pharmacy Practice \& Administration, College of Pharmacy, Ohio State University, Columbus, OH 43210, USA; Tel: (614) 688-4249; Fax: (614) 529-5070;

E-mail: Szeinbach.1@osu.edu loss of function, decreased work productivity and health outcomes [5-8]. Alternatively, the prevalence of allergic rhinitis ranges from $10 \%$ to $40 \%$ in the general population [9]. Annual costs to treat allergic rhinitis range from $2-5$ billion in the US [10] with higher costs reported when other comorbidities such as sinusitis and asthma were present [11, 12]. Respiratory diseases are known to have a profound negative impact on quality of life [13], health outcomes [14], and contribute to significant losses in work productivity and quality of life [15-18]. When sleep disorders and respiratory diseases were considered concomitantly, there were repeated observations that sleep disorders, especially obstructive sleep apnea (OSA) syndrome were major complaints associated with upper respiratory disease [19-21]. Co-morbidities have an economic impact on health care resource utilization and individual performance in the workplace as well as an impact on the patient evaluation process. The challenge for physicians, specialists, and other prescribers is to disentangle patient complaints, symptoms, and history from other clinical measures to determine treatment options that will ultimately influence patient-reported outcomes (PRO) (Fig. 1). Given these challenges, the goal of this study was to examine the relationship between sleep disorders, respiratory disease, and other co-morbidities in a group of participants with chronic constipation. An examination of co-morbidities in chronic 


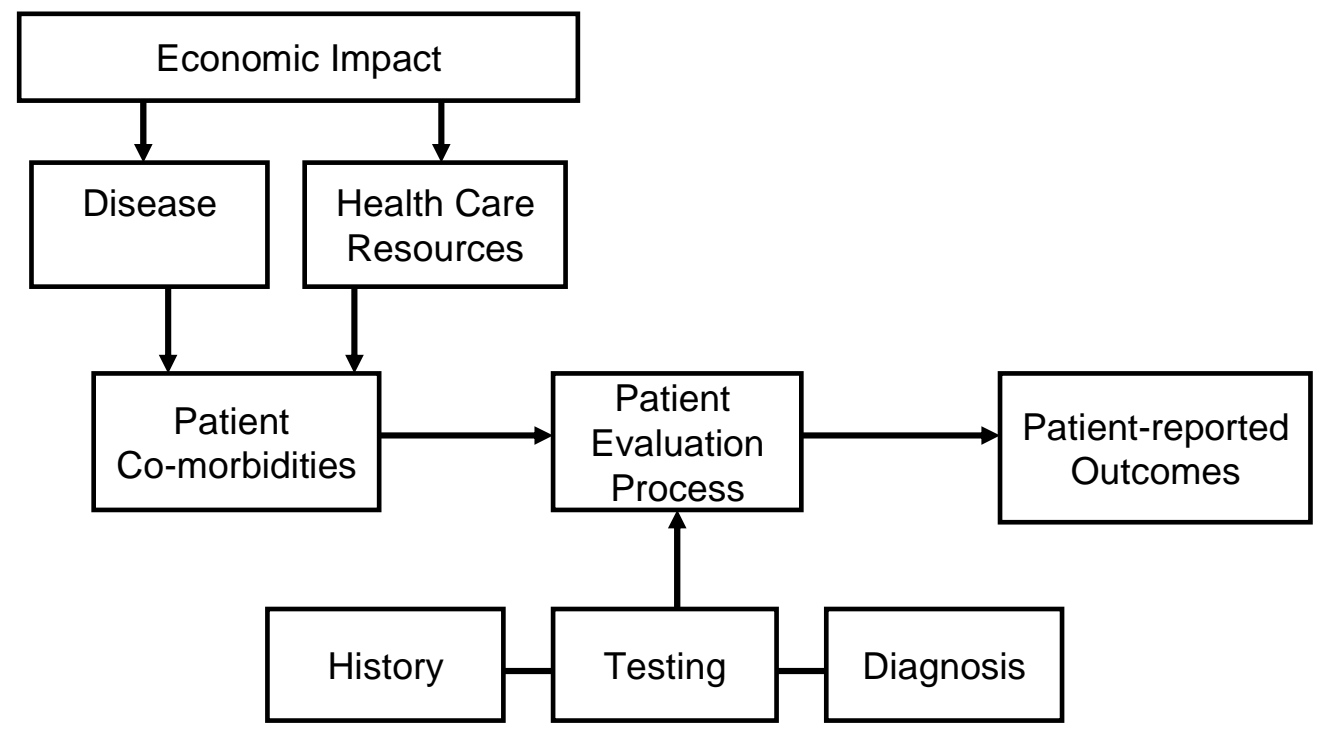

Fig. (1). Influence of co-morbidities and patient evaluation process on patient-reported outcomes.

constipation participants with and without sleep disorders may provide additional insight into the patient evaluation process, thus supporting the need for diagnostic testing when patient complaints are inconsistent with a suspected disease state.

\section{METHODS}

\section{Participants}

Harris Interactive provided an online panel consisting of several million people who agreed to participate in survey research. Panel members were obtained through 100 different sources that used a wide range of recruitment strategies such as graphical dispersion, professional meetings, and telephone recruitment to reduce selection bias. Password protected email invitations were used to control entry, maintain data integrity, and to gain access to selected survey results after completion. Prior to dissemination, the questionnaire was approved by an ethics committee at Harris Interactive, and the study protocol was approved by an Institutional Review Board from a university located in the Midwest United States. Participants confirmed their desire to enter the panel by clinking on a designated link and registering. To be included, participants had to be US citizens, 18 years of age or older, diagnosed with digestive or GI disorder by a physician, nurse, or other health-care professional, diagnosed with chronic constipation, and had to meet ROME II criteria for chronic constipation. The sample included patients with chronic constipation and patients with constipation predominated IBS (IBS-c) who reported two or more symptoms for a total 12 weeks in the past year [22]. Information elicited from participants for this portion of the study included demographics, participant characteristics, co-morbidities, health, care access and experience. Participants were also asked to rate their general health using a scale: $1=$ very poor, 2 = poor, $3=$ fair, $4=$ good, $5=$ very good, $6=$ excellent.

\section{Statistical Approach}

Data were analyzed using SPSS 16.0 (Windows (SPSS Inc., Chicago, IL). Logistic regression models were used to predict the impact of co-morbid conditions while considering age, gender, current symptom severity, and prescribed medication use. The Hosmer-Lemeshow test of model fit was used to assess the fit of binary-coded independent variables for each co-morbid condition. One-way analysis of variance (ANOVA) was used to examine the impact of co-morbidities on participant perceptions of general health. The P-value for statistical significance was 0.05 .

\section{RESULTS}

There were 31,988 email invitations disseminated to participants who met the study criteria. Of the responses, 5,554 individuals were unable to meet the criteria for inclusion and 207 started the process but failed to complete the survey. Beginning on October 10, 2007, the screening process continued until an appropriate sample size of 311 qualified participants was reached on October 30, 2007. General information for all 311 participants revealed that approximately $84 \%$ of the sample was female with the average age of 52 (SD = 11.2; range $=24-89$ ) years for all participants. Over $80 \%$ of the sample was Caucasian, had symptoms for about 19 years, and rated their overall health as fair (Mean $=3.06$; $\mathrm{SD}=$ 1.11). Gastroesophageal reflux disease (GERD) was affirmed by $66.6 \%$ of participants, GI disorders including colitis and spastic colon were affirmed by $4.2 \%$ and $16.1 \%$ of participants, respectively.

As shown in Table 1, participants were categorized according to the study variables of interest, namely, allergic disease and sleep disorders. The IBS-c group closely resembled participants with chronic constipation, yet appeared to have IBS with constipation, thus this subgroup was examined separately as a control measure. Among these 311 participants, there were $199(64.0 \%)$ with allergic rhinitis, $162(52.0 \%)$ with sleep disorders, and 170(54.7\%) with IBSconstipation. Similar response patterns among the three groups were observed for symptom severity, medication use, and overall health. The proportion of participants with comorbid conditions, odds ratios, and the impact of chronic constipation participants with and with sleep disorders on 
Table 1. Sample Characteristics $(n=311) *$

\begin{tabular}{|c|c|c|c|}
\hline Characteristic & Allergies & IBS-constipation & Sleep Disorders \\
\hline & $(n=199)$ & $(\mathrm{n}=170)$ & $(n=162)$ \\
\hline Age (mean years, SD) & $51.6(11.4)$ & $50.4(10.5)$ & $52.4(9.4)$ \\
\hline Symptoms for how long? (mean years, SD) & $19.8(16.3)$ & $19.1(15.0)$ & $18.4(16.3)$ \\
\hline Caucasian & 80.4 & 80.7 & 83.3 \\
\hline Asian or Pacific Islander & 11.1 & 10 & 9.9 \\
\hline African-American & 3 & 4.1 & 3.7 \\
\hline Prescribed medication (\% yes) & 32.7 & 34.7 & 36.4 \\
\hline Overall health $* * *$ (mean, SD) & $3.09(1.1)$ & $3.0(1.1)$ & $2.8(1.1)$ \\
\hline
\end{tabular}

* All patients sampled met ROME II criteria for chronic constipation

** Scale: $1=$ not severe at all; $10=$ extremely severe

$* * *$ Scale: $1=$ very poor $; 6=$ excellent

overall health are presented in Table 2 . Logistic regression analysis was used to evaluate the impact of co-morbid conditions and chronic constipation participants with and without sleep disorders adjusted for age, gender, current symptom severity, and prescribed medication use. Once sample characteristics were controlled, results revealed significant odds ratios for all respiratory diseases in participants with chronic constipation and sleep disorders (Table 2). Significant odds ratios were noted for other co-morbidities including chronic back problems, fibromyalgia, hair loss or thinning, incontinence, headaches, obesity, and vision impairment with respect to sleep disorders. With the exception of a significant Hosmer-Lemeshow test for arthritis and overactive bladder, the other co-morbid conditions including cholesterol problems, diabetes, endometriosis, IBS-c, heart disease hypertension, menopause, osteoporosis, skin, and thyroid conditions were not significant. A one-way ANOVA was conducted to examine the hypothesis that the mean value for overall health was lower for all constipation participants who also experienced other co-morbid conditions. Results from oneway ANOVA revealed that perceptions of overall health were significantly worse for participants with arthritis, asthma, COPD, chronic bronchitis and chronic sinusitis, fibromyalgia, chronic back pain, headaches, obesity, sleep disorders, and vision impairment. For allergies, the p-value did not reach statistical significance $(\mathrm{p}=0.09)$.

\section{DISCUSSION}

In this study, co-morbidities were examined in a population of participants having chronic constipation with or without sleep disorders. For participants with chronic constipation, the risk of having sleep disorders was higher when respiratory diseases were present, especially COPD (OR 4.0; $\mathrm{p}<0.001)$. Other co-morbid conditions that revealed a greater risk for having sleep disorders included disease states that produced discomfort and pain, obesity, incontinence, and hearing loss. All participants with chronic constipation, in general, perceived that their overall health was worse when co-morbidities including respiratory diseases, disease states with pain, sleep disorders, and obesity were present.

Considering that most patients gain access to the health care system through primary care physicians and other prescribers, the challenge for physicians is to assess the impact of co-morbid conditions through family history, patterns of medication use, and diagnostic testing. During the initial phase of patient evaluation, physicians and allergists consider the type, severity, and duration of patient-reported symptoms. Although this initial evaluation may not reduce the incidence of illness in the long run [23], at least symptoms can be documented and evaluated to determine if pharmacotherapy is an option or if referral is warranted. With a large number of individuals seeking alternative medicines and treatments, physicians and allergists must consider a broader range of agents that could trigger an allergic response. Most importantly, communication with patients at this stage is critical as some patients may be reluctant to report disorders, may be uncertain about the cause of their complaints or unable to express their concerns, and may underestimate the impact of other co-morbid conditions, thus failing to document all medications taken on the initial assessment form.

When multiple co-morbidities are suspected, early detection and confirmation of allergic disease is imperative to optimize pharmacotherapy and improve long-term patient outcomes. For respiratory diseases, the traditional pathway for diagnostic testing involves either skin testing or specific 
Table 2. Chronic Constipation Patients with and Without Sleep Disorders and Co-morbidities and Overall Health

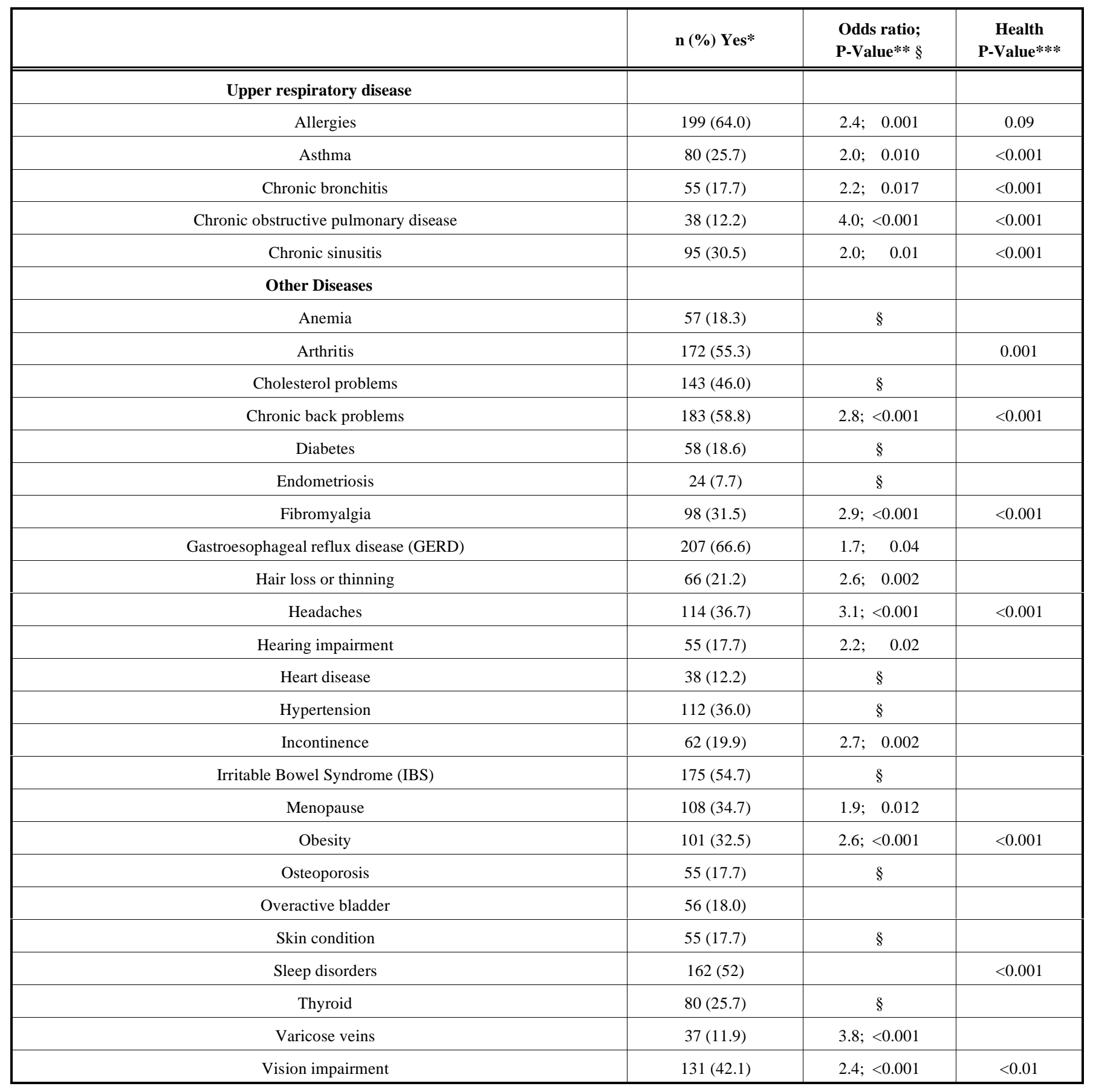

* Percentage of chronic constipation patients $(\mathrm{n}=311)$ by co-morbidities

** Logistic regression odds ratio and P-values for chronic constipation patients with and without sleep disorders by co-morbidities

$\S$ Non-significant co-morbid conditions; significant Hosmer-Lemeshow test for arthritis and overactive bladder

$* * *$ ANOVA results for co-morbidities by health $(1=$ very poor; $6=$ excellent); significant values indicate perceptions of worse health

IgE blood testing. Specific IgE blood testing may be preferred by primary care physicians because testing is relatively ease to manage and results are highly accurate [24 28]. Another test, the basophil activation test is also a highly reliable technique in the diagnosis of allergy to inhalant allergens [29]. Findings from other studies; however, posit that specific IgE blood levels may not be consistent if a patient is followed using 2 different assay systems [30]. An emerging area for research includes the topic of toll-like receptors, which appear to underlie the pathogenesis for these conditions through their ability to trigger an immune response and inflammation in respiratory disease and certain autoimmunebased gastrointestinal (GI) disorders such as ulcerative colitis and Crohn's disease [31-33]. Thus, for patients with multiple disease states, it may be beneficial to use one system to test for several allergens. 


\section{Respiratory Disease, Insomnia and Chronic Constipation}

Given the widespread symptoms associated with GI problems, sleep complaints should be expected, yet there is a paucity of studies that address the relationship between sleep disturbances and GI symptoms. Results from a retrospective analysis revealed that individuals in Olmsted County with sleep disturbances had a higher prevalence of IBS but not dyspepsia, compared to individuals reporting normal sleep [34]. In another study including over 6,000 residents in Olmsted County, the univariate association between sleep symptom score and GI symptoms increased step-wise as both symptoms worsened $(\mathrm{r}=0.28, \mathrm{p}<0.001)$ [35]. Poor sleep was also associated with increased odds for multiple upper and lower GI symptoms including abdominal pain, diarrhea, loose stools and constipation [35]. Other studies demonstrated a relationship between IBS and functional dyspepsia and sleep disturbances [36]. However, conclusions regarding the relationship between sleep disorders, IBS, chronic constipation, and related disorders needs further evaluation because pain is a predominate symptom that differentiates IBS patients with and without constipation, and this distinction among IBS patients was not always clear.

In this study the odds ratio between chronic constipation participants with sleep disorders and IBS-C participants was not significant. Although this observation may provide only minimal support that sleep disorders in this population were attributed to co-morbidities other than chronic constipation, results from this study need conformation using a more rigorous approach in a controlled environment and with a larger cohort of patients. Despite the inability to draw conclusions regarding the nature of the relationship, our findings were consistent with previous studies that examined relationships between pain and sleep [37-40], obesity, and asthma [41, 42 ]. In a previous study of patients with sudden hearing loss, authors concluded that patients who developed sudden hearing loss tended to have OSA more frequently than those individuals in the control group [43]. If several co-morbid conditions are present, patients may benefit from additional testing, evaluation, and referral, all of which impart a substantial economic burden to society, yet may be necessary to pinpoint patient complaints.

\section{Patient-reported outcomes}

PROs are increasingly used by health professionals as end points to describe the impact of treatment from the perspective of patients. Results of this study suggest that the impact of co-morbid conditions on PROs is multifaceted across disease states, moderated through individual responses, and influenced by geographical regions and environmental factors. With all diseases considered concomitantly, perhaps the greatest impact of co-morbidities is on the disease threshold, that is, the nexus between disease manifestations based on symptom severity and the presence of detectable chemical markers in the serum. Without appropriate intervention at this juncture, symptoms will likely have an adverse impact on PROs such as quality of life, medication adherence, and overall health. The importance of symptom severity in patient assessment was reinforced in a previous study of family physicians. Besides considering how well test results related to symptoms, physicians also considered symptom severity, symptom length and family history when recommending the use of specific IgE blood testing for patients suspected of having allergic rhinitis [44]. Thus, from the standpoint of diagnostic testing, treatment satisfaction and the value of treatment as perceived by patients may reside in the ability to control symptoms or, perhaps, to determine if symptoms were attributed to the suspected disease state or a specific allergen.

If referral to an allergist or other specialist is warranted, information specific to the primary diagnosis should be considered as well as secondary information that could be missed upon initial examination if medical records and patient history were not complete. Medication use and medication dosing schedules should be examined for possible interactions between diagnostic tests and medications, herbal products, and other natural remedies. As patients take more medications, pharmacokinetic parameters for drug metabolism may alter the disease threshold thereby giving an appearance of a disease state or symptoms that may inconsistent with the initial evaluation or diagnosis. In summary, the bottom-line approach needed to disentangle symptoms of upper respiratory disease from true sleep disorders should entail a two-stage strategy that includes a careful evaluation of patient history, symptoms, and a careful evaluation of current medication use. Once a baseline profile is established, the second stage of patient evaluation should encompass extensive diagnostic testing, immune response challenges, and other patient-reported assessment tools.

\section{CONCLUSIONS}

Quality of life and other PROs may be jeopardized in the presence of co-morbid conditions. Although primary care physicians and other prescribers have a traditional role as health system gatekeepers, patient evaluation and assessment becomes more challenging when patient-reported symptoms are confounded with other disease states. In these situations, allergists and other specialists need a broader approach to evaluate patients for suspected allergic disease and to determine how symptoms and pharmacotherapy may alter the disease threshold. As suggested from this study and supported in the literature, upper respiratory diseases contribute to sleep disorders primarily because of their impact on breathing, whereas pain appears to be the likely culprit underlying sleep disorders associated with other comorbidities. Moreover, patient complaints involving only a few symptoms, or a number of non-specific symptoms, may not provide an accurate representation of the underlying disease or allergic response in the presence of co-morbid conditions. In summary, as individual risk for co-morbid conditions increases, physicians may need to adjust their initial evaluation of patients to encompass a broader range of disease states and symptoms.

\section{REFERENCES}

[1] Collop N. Sleep and sleep disorders in chronic obstructive pulmonary disease. Respiration 2009 12. [Epub ahead of print]

[2] Johansson P, Alehagen U, Svanborg E, Dahlström U, Broström A. Sleep disordered breathing in an elderly community-living population: Relationship to cardiac function, insomnia symptoms and daytime sleepiness. Sleep Med 2009; 10(9): 1005-11.

[3] Parish JM. Sleep-related problems in common medical conditions. Chest 2009; 135(2): 563-72.

[4] Ohayon MM. Epidemiology of insomnia: what we know and what we still need to learn. Sleep Med Rev 2002; 6(2): 97-111. 
[5] Walsh JK, Engelhardt CL. The direct economic costs of insomnia in the United States for. Sleep 1999; 22 (Suppl 2): S386-93.

[6] Ozminkowski RJ, Wang S, Walsh JK. The direct and indirect costs of untreated insomnia in adults in the United States. Sleep 2007; 30 (3): 263-73.

[7] Chilcott LA, Shapiro CM. The socioeconomic impact of insomnia: An overview. Pharmacoeconomics 1996; 10 (Suppl) 1: 1-14.

[8] Katz DA, McHorney CA. The relationship between insomnia and health-related quality of life in patients with chronic illnesses. J Fam Pract 2002; 51: 229-35.

[9] Simoens S, Laekeman G. Pharmacotherapy of allergic rhinitis: A pharmco-economic approach. Allergy 2009; 64: 85-95.

[10] Reed SD, Lee TA, McCrory DC. The economic burden of allergic rhinitis. Pharmacoeconomics 2004; 22(6): 345-61.

[11] Dalal AA, Stanford R, Henk H, Borah B. Economic burden of rhinitis in managed care: A retrospective claims data analysis. Ann Allergy Asthma Immunol 2008; 101(1): 23-29.

[12] Yawn BP, Yunginger JW, Wollan PC, Reed CE, Silverstein MD, Harris AG. Allergic rhinitis in Rochester, Minnesota residents with asthma: Frequency and impact on health care charges. J Allergy Clin Immunol 1999; 103(1), Part: 54-9.

[13] Juniper EF, Rohrbaugh T, Meltzer EO. A questionnaire to measure quality of life in adults with nocturnal allergic rhinoconjunctivitis. $\mathbf{J}$ Allergy Clin Immunol 2003; 111: 484-90.

[14] Szeinbach SL, Williams PB, Kucukarslan S, Elhefni H. Influence of patient care provider on patient health outcomes in allergic rhinitis. Ann Allergy Asthma Immunol 2005; 95: 167-74.

[15] Szeinbach SL, Seoane-Vazquez, Beyer AP, Williams PB. The impact of allergic rhinitis on work productivity. Prim Care Res 2007; 16(2): 98-105.

[16] World Health Organization. Chronic obstructive pulmonary disease (COPD) fact sheet. Available at http: //www.who.int/mediacentre/factsheets/fs315/en/index.html; May 2008 [accessed 10.26.2009].

[17] Menzin J, Boulanger L, Marton J, et al. The economic burden of chronic obstructive pulmonary disease (COPD) in the U.S. Medicare population. Res Med 2008; 102; 1248-56.

[18] Bahadori K, Boyle-Waters MM, Marra C, et al. Economic burden of asthma: A systematic review. BMC Pulm Med 2009; 9: 24.

[19] Stull DE, Vernon MK, Canonica W, et al. Using the congestion quantifier seven-item test to assess change in patient symptoms and their impact. Allergy Asthma Proc 2008; 29(3): 295-303.

[20] Mullol J, Maurer M, Bousquet J. Sleep and allergic rhinitis. J Investig Allergol Clin Immunol 2008; 18(6): 415-9.

[21] Braig TJ, Ferguson BJ, Krouse JH. Sleep impairment in allergic rhinitis, rhino-sinusitis, and nasal polyposis. Am J Otolaryngol 2008; 29(3): 209-17.

[22] Szeinbach SL, Baran RW, Bratten J, Jones MP. Psychometric development and validation of the chronic constipation treatment satisfaction questionnaire (CTSAT-Q). Value Health 2009; 12(6): 1004-10.

[23] Picone G, Sloan F, Taylor D. Jr. Effects of risk and time preference and expected longevity on demand for medical tests. J Risk Uncertaint 2004; 28(1): 39-53.

[24] William PB, Barnes JH, Szeinbach SL, et al. Analytic precision and accuracy of commercial immunoassays for specific IgE: Establishing a standard. J Allergy Clin Immunol 2000; 105: 121-30.

[25] Szeinbach SL, Barnes JH, Sullivan TJ, et al. Precision and accuracy of commercial laboratories' ability to classify positive and/or negative allergen-specific IgE results. Ann Allergy Asthma Immunol 2001; 86: 373-81.
[26] Killingsworth LM. Specific IgE testing: Objective evidence of sensitization aids diagnosis and treatment decisions. Lab Med 2007; 38(1): 17-20.

[27] Ahlstedt S, Murray CS. In vitro diagnosis of allergy: How to interpret $\mathrm{IgE}$ antibody results in clinical practice. Prim Care Respir J 2006; 15: 228-36.

[28] Engels EA, Terrin N, Barza M, Lau J. Meta-analysis of diagnostic tests for acute sinusitis. J Clin Epid 2000; 53: 852-62.

[29] Sanz ML, Sanchez G, Gamboa PM, et al. Allergen-induced basophil activation: CD63 cell expression detected by flow cytometry in patients allergic to Dermatophagoides pteronyssinus and Lolium perenne. Clin Exp Allergy 2001; 31: 1007-13.

[30] Wang J, Godbold JH, Sampson HA. Correlation of serum allergy IgE test performed by different assay systems. J Allergy Clin Immunol 2008; 121: 1219-24.

[31] Phipps S, Lam CE, Foster PS, et al. The contribution of toll-like receptors to the pathogenesis of asthma. Immunol Cell Biol 2007; 85(6): 463-70.

[32] Lindermans C, Kimpen J, Bont L. The role of toll pathways in viral respiratory disease during early childhood. Curr Mol Med 2009; 9(5): 519-526.

[33] Himmel ME, Hardenberg G, Piccirillo CA, et al. The role of Tregulatory cells and toll-like receptors in the pathogenesis of human inflammatory bowel disease. Immunology 2008; 125(2): 14553.

[34] Vege SS, Locke GR III, Weaver AL, Farmer SA, Melton LJ III, Talley NJ. Functional gastrointestinal disorders among people with sleep disturbances: A population-based study. Mayo Clin Proc 2004; 79: 1501-6.

[35] Cremonini F, Camilleri M, Zinsmeister AR, et al. Sleep disturbances are linked to both upper and lower gastrointestinal symptoms in the general population. Neurogastroenterol Motil 2009; 21: 128-35.

[36] Wallander M, Hohansson S, Ruigomez A, et al. Dyspepsia in general practice: Incidence, risk factors, co-morbidity and mortality. Family Pract 2007; 24(5): 403-11.

[37] Maconi G, Tosetti C, Stanghellini Vm, et al. Dyspeptic symptoms in primary care. An observational study in general practice. Eur J Gastroenterol Hepatol 2002; 14: 985-90.

[38] Cappelleri JC, Bushmakin AG, McDermott AM, et al. Measurement properties of the medical study sleep scale in patients with fibromyalgia. Sleep Med 2009; 10: 766-70.

[39] Ustinov Y, Lichstein KL, Vander Wal GS, et al. Association between report of insomnia and daytime functioning. Sleep Med 2010; 11(1): 65-8.

[40] Pamuk ON, Umit H, Harmander, O. Increased frequency of gastrointestinal symptoms in patients with fibromyalgia and associated factors: A comparative study. J Rheumatol 2009; 36(8): 1720-4.

[41] van Baal PHM, Polder JJ, de Wit GA, et al. Lifetime medical costs of obesity: Prevention no cure for increasing health expenditure. PLoS Med 2008; 5(2): 242-9.

[42] Delgado J, Barranco P, Quirce S. Obesity and asthma. J Investig Allergol Clin Immunol 2008; 18(6): 420-5.

[43] Fischer Y, Yakinthou A, Mann WJ. Prevalence of obstructive sleep apnea syndrome (OSA) in patients with sudden hearing loss: A pilot study. HNO 2003; 51(6): 462-6.

[44] Szeinbach SL, Harpe SE, Williams PB, Elhefni H. Testing for allergic disease: Parameters considered and test value. BMC Family Pract 2008; 9: 47.

Received: December 14, 2009

(C) Szeinbach et al.; Licensee Bentham Open.

This is an open access article licensed under the terms of the Creative Commons Attribution Non-Commercial License (http://creativecommons.org/licenses/by-nc/3.0/) which permits unrestricted, non-commercial use, distribution and reproduction in any medium, provided the work is properly cited. 